\title{
Suicide Gene Therapy
}

National Cancer Institute

\section{Source}

National Cancer Institute. Suicide Gene Therapy. NCI Thesaurus. Code C16115.

Targeted cancer therapy that forces cancer cells to self-destruct. Suicide gene therapy involves delivering a transgene into cancer cells through various targeted delivery methods followed by systemic exposure to a pro-drug. Within target cells the pro-drug is converted to an active form by the transgene, resulting in cell death. 